\title{
Psicooncología
}

ISSN: 1696-7240

\section{Aspectos psicosociales de la calidad de vida en supervivientes pediátricos de tumores en el sistema nervioso central*}

\author{
Marta Pérez-Campdepadrós ${ }^{1, * *}$; Carmina Castellano-Tejedor ${ }^{2}$; Tomás Blasco ${ }^{3}$
}

Recibido: 3 de julio de 2018 / Aceptado: 20 de agosto de 2018

Resumen: Introducción: Los supervivientes de tumores del sistema nervioso central (TSNC) presentan peor calidad de vida en relación a la salud (CVRS) que los supervivientes de otras neoplasias y la población normativa. El tipo y gravedad de las secuelas no parecen tener un impacto claro en la CVRS, pero la afectación del cociente intelectual (CI) sí podría modular la CVRS. Además, las estrategias de afrontamiento del paciente y de los progenitores y el malestar emocional parental podrían también actuar sobre la CVRS. Objetivo: Determinar si las estrategias de afrontamiento del adolescente y su CI, y las estrategias de afrontamiento, el estrés general y el malestar emocional parental, modulan las dimensiones psicosociales de la CVRS del superviviente. Método: Estudio transversal descriptivocorrelacional. Se reclutaron 37 adolescentes (12-19 años) que llevaban $\geq 1$ año fuera de tratamiento oncológico y 44 progenitores. Resultados: En la CVRS valorada por los adolescentes, las dimensiones Relación con los Padres y Vida Familiar, Amigos y Apoyo Social, y Entorno Escolar dependen de la estrategia de afrontamiento de resolución del problema usada por el adolescente. La dimensión Aceptación Social depende del estilo de afrontamiento improductivo del adolescente y del estilo de búsqueda de ayuda social de los padres. La CVRS valorada por los padres depende de las secuelas, el CI del adolescente y, el tipo de tratamiento oncológico recibido. Discusión y conclusiones: Las estrategias de afrontamiento centradas en la resolución del problema, deben ser consideradas para valorar e intervenir en la mejora de la CVRS de los supervivientes TSNC.

Palabras clave: Tumores SNC; CVRS; supervivientes; adolescentes; CI; estrategias de afrontamiento.

1 Marta Pérez-Campdepadrós Servicio de Psiquiatría y Psicología, Hospital Sant Joan de Déu, Passeig de Sant Joan de Déu, 2, 08950 Esplugues de Llobregat, Barcelona.Servicio de Oncología y Hematología Pediátrica, Hospital Universitari Vall d'Hebron de Barcelona, Passeig de la Vall d'Hebron 119-129, 08035, Barcelona, España.

E-mail: maperez@sjdhospitalbarcelona.org

2 Carmina Castellano-Tejedor. Institut de Recerca Vall d'Hebron, Passeig de la Vall d'Hebron 119-129, 08035, Barcelona, España. Grup d’Investigació en Estrés i Salut (GIES), Departament de Psicologia Bàsica, Universitat Autònoma de Barcelona, 08193, Bellaterra, España.

E-mail: ninacastej@yahoo.es

3 Tomás Blasco. Grup d'Investigació en Estrés i Salut (GIES), Departament de Psicologia Bàsica, Universitat Autònoma de Barcelona, 08193, Bellaterra, España.

E-mail: tomas.blasco@uab.es

* Financiación: Esta investigación ha sido financiada parcialmente gracias a la ayuda económica recibida por la Asociación Española Contra el Cáncer (AECC) a través de la convocatoria de premios para la investigación del 2008 (referencia 2010.0079)

** Dirección de correspondencia: Marta Pérez Campdepadrós, Servicio Psiquiatría y Psicología. Hospital Sant Joan de Déu, Passeig de Sant Joan de Déu, 2, 08950 Esplugues de Llobregat, Barcelona, España. 


\title{
[en] Psychosocial aspects of the quality of life in pediatric survivors of tumors in the central nervous system
}

\begin{abstract}
Introduction: Central Nervous System Tumor (CNST) survivors tend to show worse HRQoL than survivors of other types of cancer and general population. There are still many doubts about what variables and to what extend these are related to their HRQoL. Type and severity of late effects have not a clear impact on HRQoL. However, Intelligence Quotient (IQ) changes as a specific late effect of this sample population, could modulate their HRQL. Furthermore, coping from both patients and parents, as well as parental distress could play a significant role modulating HRQoL in these survivors. Aim: To study whether survivors' coping strategies and IQ, as well as coping, general stress and cancerrelated distress of parents could modulate some psychosocial dimensions of their children HRQoL. Methodology: Correlational descriptive cross-sectional study. Thirty-seven teenagers (12-19 years old, both included) $\geq 1$ year free of disease and 44 parents were recruited for the study. Results: When HRQoL is assessed by adolescents, the dimensions Parent Relations and Home Life, Social Support and Peer, and School Environment are related with the use of coping with problem style by adolescents. Social Acceptance/Bullying dimension is related with nonproductive coping by the adolescent and helpseeking coping style by parents. HRQoL assessed by parents is related with late effects, adolescents' IQ, and type of oncological treatment received. Discussion/Conclusion: Coping strategies, and specifically problem-solving strategies must be taken into account to assess and to intervene in the improvement of HRQoL of this sample population.
\end{abstract}

Keywords: Central Nervous System Tumors; HRQoL; survivors; adolescence; QI; coping.

Sumario. 1. Introducción 2. Método 2.1. Diseño 2.2. Participantes 2.3. Instrumentos administrados 2.4. Procedimiento 2.5. Aspectos éticos 2.6. Análisis estadístico 3. Resultados 4. Discusión 5. Conflictos de interés 6 . Agradecimientos 7. Referencias bibliográficas.

Cómo citar: Pérez-Campdepadrós M, Castellano-Tejedor C, Blasco T. Aspectos psicosociales de la calidad de vida en supervivientes pediátricos de tumores en el sistema nervioso central. Psicooncología 2018;15:217-236. doi: 10.5209/PSIC.61432.

\section{Introducción}

Los pacientes afectados por tumores cerebrales (TC) o tumores del sistema nervioso central (TSNC), de los cuales hablaremos indistintamente en el presente estudio, tienen de forma implícita unas características diferenciadas con respecto al resto de neoplasias. Así, la integridad cerebral está en riesgo debido a la propia localización del tumor y a los efectos secundarios de los tratamientos que se aplican, como la cirugía, la radioterapia craneal (RTC) y/o quimioterapia (QT), considerándose la RTC como una de las principales causas de que aparezcan, en mayor medida que en el resto de neoplasias, secuelas a medio o largo plazo $^{(1)}$. La repercusión de estos efectos puede impedir a estos supervivientes el manejo independiente de los problemas de salud, así como dificultar su funcionamiento social autónomo ${ }^{(2)}$. Por consiguiente, su calidad de vida en relación a la salud (CVRS) puede verse gravemente afectada.

Algunos trabajos apuntan a que los déficits no se deben tanto a la pérdida de funciones, sino a la no adquisición de las mismas, lo cual justificaría encontrar los mayores déficits en aquellos supervivientes tratados en edades más tempranas o en aquellos que llevan más tiempo de supervivencia, pues el progreso evolutivo desde el punto de vista cognitivo podría haber quedado alterado ${ }^{(3,4)}$. 
La literatura que ha estudiado la relación entre CVRS y TSNC, se ha centrado mayoritariamente en adultos supervivientes de este tipo de tumores sufridos en la infancia, evaluando indicadores como el nivel educativo, el estado civil, la existencia de actividad laboral, o el estado físico y emocional general. En este sentido, los estudios disponibles muestran que, por regla general, los supervivientes de TC presentan niveles de CVRS más bajos, tanto en comparación con el resto de supervivientes de otras neoplasias ${ }^{(5-14)}$, como en comparación con la población normativa ${ }^{(3,15,16)}$.

Con el fin de conocer mejor cómo es la CVRS de estos supervivientes, diferentes estudios han tratado de dilucidar la relación entre ésta y otras variables demográficas y clínicas. En cuanto al género, algunos autores indican que no existe ninguna asociación significativa entre género y variables de $\mathrm{CVRS}^{(15)}$, pero también hay trabajos que señalan lo contrario, siendo las mujeres supervivientes de TC/TSNC las que obtienen mejores resultados en comparación con los hombres en algunos $\operatorname{aspectos}^{(17)}$.

Respecto a la edad en el momento del diagnóstico, los resultados son contradictorios. Diversos estudios señalan que los supervivientes diagnosticados de un TC a mayor edad tienden a presentar peor funcionamiento social ${ }^{(3,18)}$ y peor funcionamiento escolar. Sin embargo, Reimers y colaboradores ${ }^{(15)}$ obtienen resultados opuestos indicando que son los diagnosticados a menor edad, los que parecen mostrar mayores dificultades en establecer relaciones de amistad entre iguales y relaciones sociales en general.

En cuanto al tiempo de supervivencia transcurrido, parece existir bastante consenso en que éste se asocia negativamente con áreas de la dimensión social ${ }^{(10,19)}$ y diferentes áreas de la CVRS en general ${ }^{(3)}$. Por otro lado, los más recientemente diagnosticados parecen más preocupados por la sintomatología física que padecen, que por otros aspectos de tipo más relacional ${ }^{(15)}$.

Pese a que cabría esperar que las secuelas neuropsicológicas afectasen directamente a la CVRS, los resultados no son concluyentes. Por un lado encontramos los trabajos que no reportan relación entre la alteración de las funciones neurocognitivas y la CVRS como el de Benesch y colaboradores ${ }^{(20)}$, o el de Maddrey y colaboradores ${ }^{(4)}$; a pesar de que este último describió graves y variados déficits neuropsicológicos en su muestra de supervivientes de meduloblastomas. Opuestamente a estos resultados encontramos otros estudios que sí reportan asociaciones entre variables neuropsicológicas y CVRS. En esta línea, el deterioro en la función memorística ha sido uno de los problemas crónicos asociados negativamente con las diferentes áreas de la CVRS ${ }^{(5,21)}$. También hay estudios que sugieren que el cociente intelectual (CI) es un predictor importante de la CVRS, pues se ha indicado que este factor podría mediar los efectos que sobre la CVRS tiene la $\mathrm{RTC}^{(15)}$. Todo ello indica que son necesarios más estudios que clarifiquen el papel que juega el CI en la CVRS de los pacientes en remisión.

Junto a las variables demográficas y clínicas, las variables psicológicas tanto del propio adolescente, como de su entorno social más cercano, podrían influir o mediatizar en su CVRS en periodo de supervivencia a un TC, lo cual ha sido observado en estudios que han utilizado muestras de supervivientes de tumores no$\mathrm{TC} / \mathrm{SNC}^{(22)}$. Dentro de las variables psicológicas debe tenerse en cuenta el posible papel del entorno familiar, pues se ha sugerido que el superviviente adolescente de TC en etapa infantil puede tener problemas para realizar su proceso hacia la 
independencia de los padres, lo cual vendría avalado por el hecho de que este grupo de supervivientes vive menos frecuentemente en pareja y tiene mayor incidencia de situación de desempleo ${ }^{(7)}$. Esta dependencia, debida a la presencia de efectos a largo plazo puede impactar física y emocionalmente a los progenitores o cuidadores principales ${ }^{(23)}$. En esta línea, los datos disponibles muestran que un $56 \%$ de progenitores reportan unos altos niveles de malestar emocional general ${ }^{(23)}$, y un $29 \%$ muestran niveles graves de sintomatología de estrés postraumático ${ }^{(24)}$, e incluso algunos autores describen un porcentaje del $42 \%$ de progenitores que cumplen criterios diagnósticos de estrés de tipo postraumático según criterios DSM-IV ${ }^{(24)}$. Además, el malestar emocional parental persiste también durante el período de supervivencia ${ }^{(26-28)}$. En consecuencia, cabe preguntarse si este malestar emocional de los padres y las estrategias de afrontamiento que puedan utilizar para manejarlo podrían estar también modulando la CVRS del superviviente, tal como se ha sugerido en algún estudio ${ }^{(22)}$.

Vemos que, si bien parece estar claro que los supervivientes de TC tienden a presentar peor CVRS que el resto de supervivientes de otras neoplasias y que la población normativa, existen muchas dudas sobre qué variables pueden modular determinadas dimensiones de la CVRS y en qué medida lo hacen. Dentro de esta problemática es importante fijarse especialmente en los aspectos psicosociales de la CVRS, ya que, como hemos señalado antes, estos pueden determinar en buena medida la capacidad del adolescente para independizarse de los padres. Por lo que identificar las variables que puedan afectar a los aspectos psicosociales podría ser relevante para la mejora de la CVRS. Los datos de que se dispone no permiten dar respuesta a esta cuestión, pero inducen a sospechar que el CI y las estrategias de afrontamiento del adolescente pueden jugar un papel clave, junto con las reacciones que los padres muestran ante la situación de supervivencia.

En base a todo lo descrito, el presente trabajo tiene como objetivo analizar si las dimensiones psicosociales de la CVRS del adolescente, están moduladas por las estrategias de afrontamiento y el CI del propio adolescente, y por las estrategias de afrontamiento, el estrés general, y el malestar emocional parental.

\section{Método}

\subsection{Diseño}

Se trata de un diseño descriptivo correlacional de tipo transversal.

\subsection{Participantes}

\section{Adolescentes}

La muestra final estuvo constituida por 37 adolescentes oncológicos en remisión de entre 12 y 19 años de edad (ambos incluidos) cuyas características se describen en la Tabla 1 .

Los criterios de inclusión fueron: 1) haber sido diagnosticado de un TC/TSNC entre los 0 y los 18 años, 2) tener entre 12 y 19 años (ambos incluidos) en el momento del estudio, 3) llevar fuera de tratamiento oncológico un período de tiempo igual o 
superior a 1 año, 4) ser capaz de comprender y hablar castellano o catalán, y 5) tener programada una visita de seguimiento en el hospital en los meses en que se llevó a cabo la recogida de datos para la presente investigación. Como criterios de exclusión se estableció: 1) el presentar psicopatología y/o alteración neurológica, previa al diagnóstico de la enfermedad oncológica y sin relación con la misma, o 2) tener otras neoplasias, o enfermedades relacionadas, tanto previas como posteriores al diagnóstico de TC/TSNC.

Tabla 1. Características demográficas y clínicas de la muestra de adolescentes oncológicos en remisión ( $\mathrm{N}=37)$.

\begin{tabular}{|c|c|c|c|}
\hline \multirow{2}{*}{\multicolumn{4}{|c|}{ Género }} \\
\hline & & & \\
\hline Hombre & \multicolumn{2}{|c|}{21} & 56,8 \\
\hline Mujer & \multicolumn{2}{|c|}{16} & 43,2 \\
\hline \multicolumn{4}{|l|}{ Diagnóstico } \\
\hline Meduloblastoma & \multicolumn{2}{|c|}{7} & 18,9 \\
\hline Astrocitoma & \multicolumn{2}{|c|}{14} & 32,4 \\
\hline Craniofaringioma & \multicolumn{2}{|c|}{6} & 16,2 \\
\hline Ependimoma & \multicolumn{2}{|c|}{1} & 2,7 \\
\hline Tumor de tronco & \multicolumn{2}{|c|}{1} & 2,7 \\
\hline Otros & \multicolumn{2}{|c|}{8} & 21,6 \\
\hline \multicolumn{4}{|l|}{ Tratamiento } \\
\hline Intervención quirúrgica & \multicolumn{2}{|c|}{36} & 97 \\
\hline Radioterapia & \multicolumn{2}{|c|}{25} & 68 \\
\hline Quimioterapia & \multicolumn{2}{|c|}{14} & 38 \\
\hline \multicolumn{4}{|l|}{ Válvula de derivación ventrículo-peritoneal } \\
\hline 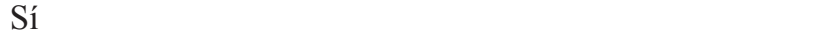 & \multicolumn{2}{|c|}{21} & 56,7 \\
\hline No & \multicolumn{2}{|c|}{16} & 43,2 \\
\hline \multicolumn{4}{|l|}{ Trasplante de médula ósea } \\
\hline Sí (autólogo) & \multicolumn{2}{|c|}{2} & 5,3 \\
\hline No & \multirow{2}{*}{\multicolumn{2}{|c|}{35}} & 94,6 \\
\hline \multicolumn{2}{|l|}{ Recidiva } & & \\
\hline Sí & \multirow{2}{*}{\multicolumn{2}{|c|}{$\begin{array}{c}6 \\
31 \\
\end{array}$}} & 16,2 \\
\hline \multirow[t]{2}{*}{ No } & & & 83,8 \\
\hline & Media & DT & Rango \\
\hline Edad en el momento de evaluación. & 15,70 & 2,45 & $12-18$ \\
\hline Edad en el momento del diagnóstico en años. Media (SD) & 7,11 & 3,77 & $1-17$ \\
\hline Años transcurridos desde el diagnóstico. & 8,70 & 4,04 & $1-18$ \\
\hline
\end{tabular}

La obtención de la muestra se realizó a partir del Registro Nacional de Tumores Infantiles (RNTI) y de un registro propio del Servicio de Oncología y Hematología Pediátricas del Hospital Universitario Vall d'Hebron de Barcelona, que facilitaron los datos de posibles participantes según los criterios de inclusión y exclusión establecidos. El periodo de reclutamiento y recolección de datos tuvo lugar entre 
abril del 2009 y diciembre del 2011. Se obtuvieron 49 potenciales participantes, excluyéndose a 12 de ellos $(24,5 \%)$ por los motivos siguientes: 6 adolescentes ( 3 chicos y 3 chicas) no fueron localizados previamente a su visita médica, 4 casos ( 1 chico y 3 chicas) rechazaron participar, 1 chico fue incapaz de responder a los cuestionarios a causa de problemas de ansiedad y, finalmente 1 chico estaba citado para el estudio pero no se presentó.

\section{Progenitores o cuidadores principales}

La muestra total de progenitores y/o cuidadores principales $(\mathrm{N}=44)$ estuvo constituida por 33 mujeres $(75 \%)$ y 11 hombres $(25 \%)$. Su media de edad fue de 48,25 años ( $\mathrm{DT}=10,10$; rango 36-60 años). Todos ellos madres o padres respectivamente, a excepción de una abuela que era la cuidadora principal del paciente. Respecto al nivel de estudios, un 2,4\% no tenían estudios pero sabían leer y escribir, un 9,5\% tenían un nivel de estudios inferior a graduado escolar, un 21,4\% habían finalizado el graduado escolar, un 35,7\% el bachiller superior, BUP, FP o similares, un 30,9\% habían cursado estudios universitarios y un $2,4 \%$ no respondieron a esta pregunta. La situación laboral de los padres era de jornada completa en un $47,4 \%$ y de media jornada en un $15,9 \%$. También se observó un $20,5 \%$ de amas de casa, y un $4,5 \%$ en paro, otro $4,5 \%$ jubilados y también, un $4,5 \%$ incapacitados. En un caso $(2,2 \%)$ no existía progenitor puesto que había fallecido.

Para el análisis de datos se ha considerado un único progenitor/cuidador para cada superviviente, eligiéndose a la madre en aquellos casos en los que hubieran respondido los dos progenitores, por lo que la muestra final estaba compuesta por 32 madres, 1 abuela, y 4 padres.

\subsection{Instrumentos administrados}

\section{Adolescentes}

\section{Datos sociodemográficos y médicos}

Los datos sociodemográficos y médicos se obtuvieron siguiendo un protocolo específico desarrollado ad hoc para la presente investigación, a partir de los conocimientos teóricos existentes sobre efectos tardíos y secuelas en población pediátrica superviviente a un $\mathrm{TC} / \mathrm{TSNC}^{(28)}$. Dicho protocolo se administró en formato de entrevista semi-estructurada en presencia del adolescente y su(s) progenitor(es), siendo completada y revisada la información con datos de las historias clínicas de los pacientes.

Además de la presencia o no de secuelas, recogimos la gravedad de las mismas. Debido a la inexistencia de un protocolo estándar de uso generalizado para la cuantificación de dicha gravedad por tipo de secuelas en esta población, se elaboró un índice propio para la presente investigación basado en el Late Effects Severity Score (LESS), descrito en el estudio de Benesch et al. ${ }^{(20)}$. Este nuevo índice denominado Late Effects Severity Index-Central Nervous System Tumours (LESI-CNS), valora la presencia y gravedad de secuelas y/o alteraciones en 5 áreas: 1. Neurológica, 2. Endocrina, 3. Visual/auditiva, 4. Cognitiva y 5. Otras. Cada categoría recibe $0,1 \mathrm{o}$ 
2 puntos, en función de la gravedad de la alteración. Para la presente investigación, las secuelas serán consideradas como variables dicotómicas. Así, para cada una de las secuelas, los valores " 0 " serán considerados como "ausencia de la secuela" y los valores "1" o "2", como "presencia de la secuela".

\section{Calidad de vida en relación a la salud (CVRS)}

Se utilizó el cuestionario Kidscreen-52 $2^{(30)}$ en versión auto-informada (versión auto) y en la adaptación española ${ }^{(31)}$. El Kidscreen consta de 52 ítems agrupados en 10 dimensiones: 1. Bienestar físico, 2. Bienestar psicológico, 3. Estado de ánimo y emociones, 4. Auto-percepción, 5. Autonomía, 6. Relación con los padres y vida familiar, 7. Amigos y apoyo social, 8. Entorno escolar, 9. Aceptación social (ausencia de bullying) y 10. Recursos económicos. El rango de puntuación de todos los ítems es de 1 a 5 y para cada dimensión se calcula una puntuación normalizada con un valor medio de 50 y una puntuación típica de 10. Cuenta con baremos europeos para edades comprendidas entre los 8 y 18 años, y también en función del género. Este cuestionario ha demostrado ser un instrumento útil y válido tanto en población pediátrica general, como en población pediátrica afecta de distintas enfermedades crónicas y/o con problemas de salud. Para los resultados del presente artículo, se utilizaron solamente las dimensiones 6, 7, 8 y 9, que son las que valoran los aspectos psicosociales de la CVRS.

\section{Estrategias de afrontamiento}

Se administró la Adolescent Coping Scale (en adelante, ACS) de Frydenberg y Lewis $^{(32,33)}$, en su adaptación española de Pereña y Seisdedos ${ }^{(34)}$. Este inventario de auto-informe consta de 80 ítems a partir de los cuáles se obtiene información acerca de la frecuencia de uso de 18 estrategias de afrontamiento. De los 80 elementos de los que se compone el instrumento, un ítem es abierto y los 79 restantes son de respuesta cerrada en una escala Likert de 5 puntos (de $1=$ No me ocurre nunca o no lo hago, a $5=$ Me ocurre o lo hago con mucha frecuencia). Las 18 estrategias de afrontamiento pueden agruparse en tres estilos básicos que serán los utilizados en la presente investigación: 1. Afrontamiento dirigido a la resolución del problema, 2. Afrontamiento en relación con los demás y 3 . Afrontamiento improductivo ${ }^{(32-34)}$. Para cada uno de ellos se establecen unos puntos de corte que determinan la frecuencia de uso de cada estilo en cinco categorías: nunca, raras veces, algunas veces, a menudo, con mucha frecuencia.

\section{Funcionamiento cognitivo}

Se utilizaron las Escalas Wechsler que son una batería de pruebas destinadas a valorar el funcionamiento cognitivo de un sujeto ${ }^{(35-38)}$. Para el presente trabajo se han seleccionado los subtests que proporcionan los cuatro CI correspondientes a: 1 . Comprensión verbal (CV), 2. Razonamiento perceptivo (RP), 3. Memoria de trabajo (MT) y 4. Velocidad de procesamiento (VP), con el objetivo de, por un lado, valorar qué campos son los más afectados y, por otro lado, si la disfunción de un área tiene mayor impacto en comparación con las otras sobre la CVRS. Por consiguiente, de la versión infantil WISC-IV se administraron los siguientes subtests: cubos, 
semejanzas, conceptos, dígitos, claves, vocabulario, letras y números, matrices, comprensión y símbolos. En la versión de adultos WAIS-III se administraron: cubos, semejanzas, dígitos, claves, vocabulario, aritmética, matrices, símbolos, información y letras y números. En cada una de las capacidades cognitivas se categorizó al participante en uno de estos dos niveles: CI bajo (inferior a 85) y CI medio/alto (igual o superior a 85).

\section{Progenitores y/o cuidadores principales}

\section{CVRS del adolescente}

Se administró el Kidscreen-52 versión para padres (versión proxy). Las dimensiones evaluadas así como el tipo y número de ítems, son los mismos que los descritos para el caso de los adolescentes en la versión auto. La única diferencia era el tipo de enunciado previo a cada apartado o dimensión evaluada, en el que se instaba al progenitor o cuidador principal a que pensase en su propio hijo antes de responder.

\section{Estrategias de afrontamiento}

Se administró la adaptación española del $\mathrm{COPE}^{(39)}$ en su versión disposicional. Es decir, la manera general de enfrentarse a los problemas y/o situaciones estresantes que puedan acontecer. El COPE consta de 60 ítems de respuesta tipo Likert, que discrimina la frecuencia con la que el/la participante utiliza cada conducta (1. En absoluto, 2. Un poco, 3. Bastante, 4. Mucho). En el presente trabajo nos hemos basado en la clasificación propuesta por Gutiérrez et al. ${ }^{(40)}$ que establece tres estilos de afrontamiento principales: 1. Engagement (o vinculación con el problema), 2. Disengagement (o desvinculación del problema) y 3. Help-seeking (o estrategias de afrontamiento interpersonales).

\section{Estrés general percibido}

Se utilizó la Perceived Stress Scale ${ }^{(41)}$ en su adaptación española ${ }^{(42)}$. La Escala de Estrés Percibido (en adelante, PSS) evalúa el grado en que los participantes valoran las situaciones del último mes como impredecibles y fuera de control. La PSS consta de 14 ítems de respuesta en escala Likert de 5 puntos (de $0=N u n c a$, hasta 4 - Siempre). El rango de puntuación varía entre 0 (mínimo estrés percibido) y 56 (máximo estrés percibido). Se recomienda emplear un valor de 30 como punto de corte para considerar la existencia de estrés problemático.

\section{Escalas de malestar emocional a raíz de la experiencia oncológica}

Este aspecto se evaluó mediante dos ítems con respuesta tipo Likert, con 5 opciones de respuesta y que habían sido utilizados en otra investigación con adolescentes supervivientes de tumores no-SNC ${ }^{(43)}$. El primero de ellos está destinado a valorar el grado de preocupación actual respecto a la enfermedad que padeció su hijo/a (0.Nada en absoluto, 1. Un poco, 2. Bastante, 3. Mucho, 4. Muchísimo). El segundo ítem pretende valorar la frecuencia de pensamiento sobre la enfermedad en la actualidad (0. Nunca, 1. Casi nunca: alrededor de una vez al mes, 2. Alguna vez: varias veces 
por semana, 3. Muchas veces: 1 vez al día, 4. Muchísimo: varias veces al día). Esto permite completar la información aportada por el PSS, que es de carácter general y que no distingue entre el malestar provocado por la enfermedad del hijo o por otras causas.

\subsection{Procedimiento}

La evaluación se llevó a cabo en el Servicio de Oncología y Hematología en la Infancia y Trasplante de Progenitores Hematopoyéticos Pediátrico del Hospital Universitario Vall d'Hebron de Barcelona. La evaluación constaba de dos sesiones de unos 50 minutos de duración cada una para el adolescente, y una única sesión de entre 30-45 minutos para los progenitores. Las visitas de evaluación se hicieron coincidir con las visitas de seguimiento de los chicos/as que fueron diagnosticados de TC/TSNC. La primera visita de seguimiento solía tener por objetivo realizar alguna prueba de imagen, y la segunda recoger resultados con el médico oncólogo pediatra. De este modo, unos días antes de que el chico/a tuviera la primera visita programada, se contactaba telefónicamente con la familia y se les explicaba el proyecto de investigación. Si acordaban participar se les citaba el mismo día de la primera visita de seguimiento a ambos, adolescente y progenitor, y un segundo día intentando coincidir con la segunda visita de seguimiento, con el adolescente. Esto les facilitaba la participación pues no todos ellos vivían en las proximidades del hospital.

En la primera sesión se les explicaba nuevamente el proyecto y se firmaban los consentimientos informados. Se cumplimentaba el cuestionario de datos biográficos y secuelas con ambos, progenitores y superviviente, al mismo tiempo. Posteriormente, se explicaban verbalmente y por escrito las instrucciones que debían seguir los padres para poder rellenar de forma independiente y auto-administrada los cuestionarios (en un espacio diferente al del hijo/a). Al final había la posibilidad de preguntar y resolver las dudas que hubieran surgido durante la cumplimentación de los cuestionarios. Una vez entregado el dossier que debía cumplimentar el progenitor/tutor, la investigadora, en un despacho aparte, explicaba al superviviente las diferentes consignas para proceder a la evaluación. El Kidscreen-52 y el ACS eran administrados en este orden y, una vez terminados, se iniciaba la administración de la escala Wechsler, en el orden recomendado por el mismo test. Las pruebas de las subescalas que no podían administrarse en esta primera sesión, se administraban en la segunda sesión hasta completar la aplicación completa de la prueba de Weschler. Dos adolescentes de la muestra no acudieron a la segunda sesión de evaluación, por lo que la $\mathrm{n}$ en referencia la $\mathrm{CI}$ es de 35. Quince participantes fueron evaluados con el WAIS-III y 20 con el WISC-IV.

\subsection{Aspectos éticos}

Tanto el procedimiento como las medidas del estudio fueron aprobadas por el Comité de Ética del Hospital Universitario Vall d'Hebron de Barcelona. En todos los casos la participación fue voluntaria. A través de los consentimientos informados se aseguró la confidencialidad y el anonimato de los datos. Los consentimientos informados de los adolescentes fueron firmados por ellos mismos y por un progenitor/tutor en el caso de menores de 18 años. 


\subsection{Análisis estadístico}

Para analizar la prevalencia de las distintas características socio-demográficas y clínicas de la muestra, así como para describir las puntuaciones en las cuatro dimensiones del Kidscreen-52 consideradas en el presente trabajo, se utilizaron análisis descriptivos (frecuencias, porcentajes, medias, desviaciones típicas, rangos y medianas). Para la identificación de las posibles variables explicativas, se seleccionaron aquellas que mostraban una relación con cada una de las cuatro variables criterio (mediante correlación bivariada de Pearson en el caso de las variables explicativas continuas, o mediante la prueba de comparación de medias de t Student en el caso de las variables explicativas dicotómicas). Esta selección se ha hecho por separado para las versiones auto y proxy. Para explorar y cuantificar la posible relación entre las distintas variables criterio (dimensiones de CVRS) y las variables explicativas propuestas, se ha utilizado el análisis de regresión lineal múltiple con el método de pasos sucesivos (stepwise), en cada una de las dimensiones en las que había más de una variable candidata a formar parte del modelo. En aquellas dimensiones en las que sólo una variable era candidata a formar parte del modelo se ha utilizado el método "introducir" para poder obtener un valor de $\mathrm{R}^{2}$. En todos los casos, las variables dicotómicas han sido consideradas como variables dummy. La significación de todas las pruebas se consideró a un nivel de probabilidad del $5 \%$ o inferior, indicando siempre la significación exacta que ofrece el paquete estadístico SPSS. No obstante, no se consideraron relevantes para el objeto del presente estudio aquellos modelos de regresión en los que, aun cuando se alcance la significación estadística, el porcentaje de varianza explicado sea inferior al $10 \%$.

\section{Resultados}

La Tabla 2, nos muestra los resultados de las dimensiones de la CVRS del Kidscreen-52 consideradas en el presente análisis; tanto las referidas por los adolescentes (versión auto), como por los progenitores (versión proxy). Se puede observar que de forma global, las puntuaciones medias obtenidas tanto por los adolescentes como por los progenitores, se encuentran alrededor de la media poblacional $(50 \pm 10)$.

Tabla 2. Descriptivos de las dimensiones psicosociales de CVRS (Kidscreen-52, versión "auto" y "Proxy")

\begin{tabular}{lcccc}
\hline \multicolumn{1}{c}{ Dimensión CVRS } & Versión & Media (DT) & $\begin{array}{c}\text { Rango } \\
\text { (Min - Máx) }\end{array}$ & Mediana \\
\hline Relación con los padres y vida & Auto & $49,74(9,79)$ & $30,18-65,85$ & 51,81 \\
familiar & Proxy & $53,02(10,62)$ & $8,28-63,16$ & 52,12 \\
\hline \multirow{2}{*}{ Amigos y apoyo social } & Auto & $49,95(12,91)$ & $9,40-71,46$ & 50,23 \\
& Proxy & $43,05(15,14)$ & $27,81-72,50$ & 46,43 \\
\hline \multirow{2}{*}{ Entorno escolar } & Auto & $51,65(7,14)$ & $36,77-65,94$ & 50,36 \\
& Proxy & $49,07(9,57)$ & $36,77-65,94$ & 49,74 \\
\hline Aceptación social (ausencia de & Auto & $46,95(11,37)$ & $24,99-58,85$ & 42,19 \\
bullying) & Proxy & $43,53(13,75)$ & $18,25-58,83$ & 44,82 \\
\hline
\end{tabular}


La Tabla 3 presenta los estilos de afrontamiento disposicionales utilizados por los adolescentes de la muestra. El más utilizado es el de resolución del problema, seguido del afrontamiento en relación con los demás. En ambos casos, los supervivientes manifestaban hacer uso de ellos algunas veces. En cambio, el estilo de afrontamiento improductivo era usado raras veces.

Tabla 3. Descriptivos de los estilos de afrontamiento utilizados por los adolescentes, expuestos en orden descendiente según su utilización $(\mathrm{N}=37)$.

\begin{tabular}{lccc}
\hline Estilos de afrontamiento & Media (DT) & Rango (Min - Máx) & Mediana \\
\hline Resolución de problemas & $68,78(13,16)$ & $27,80-88,60$ & 60,80 \\
Relación con los demás & $54,53(9,43)$ & $31,67-91,33$ & 59,67 \\
Improductivo & $48,05(9,43)$ & $30,71-66,71$ & 36,00 \\
\hline
\end{tabular}

En la Figura 1 se indica el porcentaje de casos con alteración en cada una de las funciones cognitivas. Tal y como puede observarse, en todas las dimensiones hay más de un $50 \%$ de la muestra que presenta un CI bajo.

Figura 1. Porcentaje de casos con alteración en cada una de las funciones cognitivas evaluadas a partir de las escalas Wechsler $(\mathrm{N}=35)$.

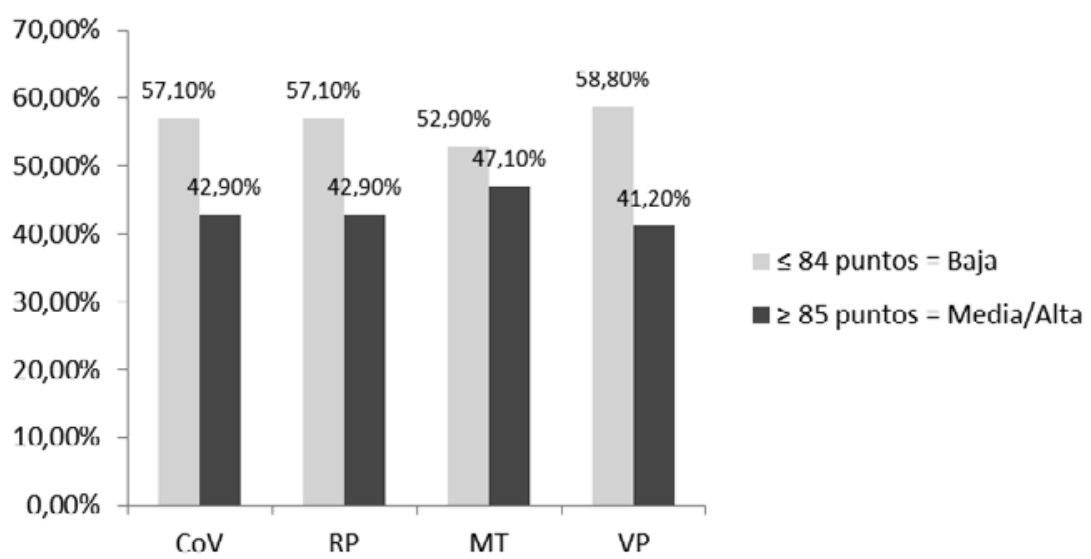

CoV: Comprensión verbal; RP: Razonamiento perceptivo; MT: Memoria de trabajo; VP: Velocidad de procesamiento.

En la Tabla 4 se describen los estilos de afrontamiento de los progenitores; siendo el más frecuentemente utilizado el de vinculación con el problema (engagement).

Respecto al estrés general de los progenitores, éste, valorado con el PSS, se sitúa en un rango observado de 5 a 42 puntos respecto a un rango posible de 0 a 56 . $\mathrm{La}$ puntuación media de estrés actual percibido en la muestra de padres es de 21,76 $(\mathrm{DT}=8,66)$ y sólo un $20,6 \%(\mathrm{n}=7)$ de los progenitores muestra sintomatología de estrés (puntuación $>29$ puntos). 
Tabla 4. Descriptivos de los estilos de afrontamiento de los progenitores $(\mathrm{N}=37)$

\begin{tabular}{lccc}
\hline Estilo de afrontamiento & Media (DT) & $\begin{array}{c}\text { Rango } \\
\text { (Min - Máx) }\end{array}$ & Mediana \\
\hline Engagement/Vinculación con el problema & $2,71(0,36)$ & $2,04-3,42$ & 2,75 \\
Helpseeking/Interpersonal & $2,40(0,51)$ & $1,50-3,58$ & 2,25 \\
Disengagement/Desvinculación del problema & $1,65(0,32)$ & $1,00-2,13$ & 1,75 \\
\hline
\end{tabular}

En la Figura 2 se recogen los diferentes grados de preocupación de los progenitores, pudiéndose observar que más de un 66\% de ellos (agrupando los valores "bastante", "mucho" y "muchísimo" muestra una preocupación elevada. En cuanto a la frecuencia de pensamiento (Figura 3), el 27,8\% no se preocupaba casi nunca (alrededor de una vez al mes); esto nos lleva a un elevado porcentaje de progenitores $(72,2 \%)$ que manifiesta seguir preocupándose alguna vez, mucho o muchísimo y que piensan en todo ello con relativa frecuencia en su día a día.

Figura 2. Grado de preocupación actual de la muestra de progenitores

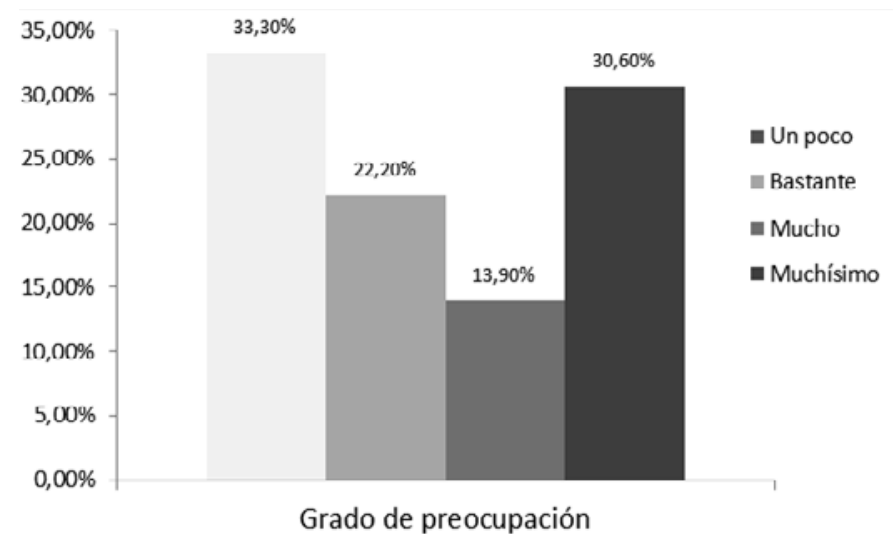

Figura 3. Frecuencia de pensamiento actual de la muestra de progenitores o cuidadores principales $(\mathrm{N}=37)$.

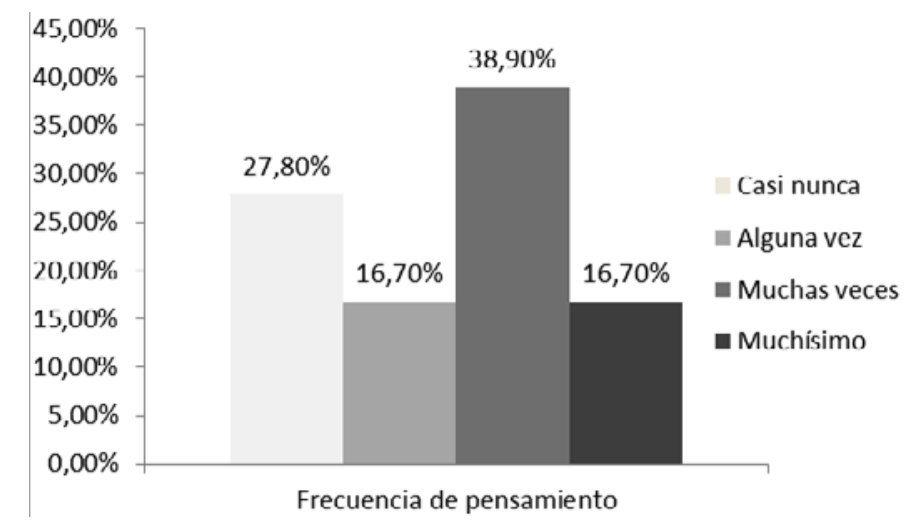


La Tabla 5, muestra los modelos de regresión obtenidos para la versión auto del Kidscreen-52. Tal y como se observa, tres modelos de regresión están relacionados con dos variables explicativas y uno con una variable explicativa. En su conjunto, comprenden un porcentaje de varianza explicada (asumiendo el valor de $\mathrm{R}^{2}$ corregida) de entre el $16,5 \%$ al $35,5 \%$. De mayor a menor varianza explicada encontramos: relación con los padres y vida familiar $\left(\mathrm{F}_{(2,34)}=10,36 ; \mathrm{p}<0,001\right)$, amigos y apoyo social $\left(\mathrm{F}_{(2,35)}=9,22 ; \mathrm{p}=0,001\right)$, aceptación social $\left(\mathrm{F}_{(2,33)}=6,32 ; \mathrm{p}=0,005\right)$ y entorno $\operatorname{escolar}\left(\mathrm{F}_{(1,34)}=7,73 ; \mathrm{p}=0,009\right)$.

Tabla 5. Resumen de los modelos de regresión lineal múltiple con coeficientes estandarizados sobre las dimensiones psicosociales del Kidscreen-52 (auto).

\begin{tabular}{cccc}
\hline Modelos de regresión estandarizados & $\mathbf{R}^{2}$ & $\mathbf{R}^{2}$ corr. & p \\
\hline $\begin{array}{c}\text { Relación con los padres y vida familiar }=(0,466 \\
\text { Estilo de afrontamiento de resolución del problema) } \\
+(0,388 \cdot \text { Edad diagnóstico })\end{array}$ & $39,3 \%$ & $35,5 \%$ & $<0,001$ \\
\hline $\begin{array}{c}\text { Amigos y apoyo social }=(0,400 \cdot \text { Estilo de } \\
\text { afrontamiento de resolución del problema })+(-0,380 \\
\text { - años transcurridos })\end{array}$ & $35,9 \%$ & $32,0 \%$ & 0,001 \\
\hline $\begin{array}{c}\text { Entorno escolar }=(0,436 \cdot \text { Estilo de afrontamiento } \\
\text { de resolución del problema })\end{array}$ & $19 \%$ & $16,5 \%$ & 0,009 \\
\hline $\begin{array}{c}\text { Aceptación social }=(-0,384 \cdot \text { Estilo de } \\
\text { afrontamiento improductivo })+(0,378 \cdot \text { Estilo de } \\
\text { afrontamiento helpseeking) }\end{array}$ & $29 \%$ & $24,4 \%$ & 0,005 \\
\hline
\end{tabular}

Finalmente los modelos de regresión obtenidos para la versión proxy del Kidscreen-52 se exponen en la Tabla 6. No hay modelo de regresión para la dimensión aceptación social. De mayor a menor varianza explicada encontramos: amigos y apoyo social $\left(\mathrm{F}_{(1,34)}=12,03 ; \mathrm{p}=0,015\right)$ y entorno escolar $\left(\mathrm{F}_{(1,34)}=7,25 ; \mathrm{p}=0,011\right)$. Los resultados de la dimensión de relación con los padres y vida familiar, pese a alcanzar la significación estadística, se han desestimado ya que ofrecen una varianza explicada inferior al $10 \%$.

Tabla 6. Resumen de los modelos de regresión lineal múltiple con coeficientes estandarizados sobre las dimensiones psicosociales del Kidscreen-52 (proxy).

\begin{tabular}{cccc}
\hline Modelos de regresión estandarizados & $\mathbf{R}^{2}$ & $\mathbf{R}^{2}$ corr. & $\mathbf{p}$ \\
\hline $\begin{array}{c}\text { Relación con los padres y vida familiar } \\
=(0,326 \cdot \text { Radioterapia })\end{array}$ & $10,6 \%$ & $8,1 \%$ & 0,049 \\
\hline $\begin{array}{c}\text { Amigos y apoyo social }=(0,419 \cdot \text { Estilo de afrontamiento de } \\
\text { resolución del problema })+(-0,336 \cdot \text { Secuelas neurológicas }) \\
+(-0,325 \cdot \text { años transcurridos })\end{array}$ & $53,8 \%$ & $49,3 \%$ & 0,015 \\
\hline \begin{tabular}{c} 
Entorno escolar $=(0,425 \cdot$ CI Velocidad de procesamiento $)(*)$ \\
\hline
\end{tabular} & $18 \%$ & $15,5 \%$ & 0,011 \\
\hline
\end{tabular}

(*) Para este modelo la muestra fue de $\mathrm{N}=35$. 


\section{Discusión}

El presente trabajo tenía por objetivo estudiar si las estrategias de afrontamiento y el CI del adolescente, y las estrategias de afrontamiento, el estrés general y el malestar emocional parental a raíz del TC sufrido por el/la hijo/a, modulan las dimensiones psicosociales de la CVRS de este/a último/a.

Según nuestros resultados, son las estrategias de afrontamiento del adolescente basadas en el estilo de resolución de problemas las que determinan en mayor medida los aspectos psicosociales de la CVRS cuando ésta es valorada por el propio adolescente. Esto concuerda, en líneas generales, con lo reportado por la literatura que ha valorado a población pediátrica superviviente a cualquier neoplasia ${ }^{(13,44-47)}$. Si bien la forma de evaluar el afrontamiento difiere ligeramente en función del instrumento utilizado, los resultados convergen en señalar que las estrategias basadas en el uso de elementos que aportan control sobre la situación, y que serían similares al estilo de resolución de problemas, se relacionan con mejores niveles de vida en los aspectos psicológicos ${ }^{(44)}$, mientras que el uso del afrontamiento improductivo, que sería lo opuesto a un estilo de resolución de problemas, guarda una relación inversa con la dimensión Amigos y Apoyo Social ${ }^{(22)}$.

Las consideraciones anteriores llevan a establecer que las relaciones con los padres, amigos, y entorno escolar, y la existencia de apoyo social pueden depender en buena medida de que el adolescente utilice un estilo de afrontamiento basado en la resolución de problemas, lo cual quedaría corroborado, según nuestros resultados, por el hecho de que en la versión proxy los padres ofrecen un resultado similar en lo relativo a las relaciones con los amigos y la existencia de apoyo social. Ahora bien, nuestros resultados no confirman que la existencia de amigos y apoyo social se vean afectados negativamente por las estrategias de afrontamiento de los padres. Sin embargo, sí hay estudios ${ }^{(22)}$ que indican que los padres pueden determinar una menor calidad de vida en esa dimensión psicosocial si utilizan un estilo de afrontamiento basado en la vinculación, posiblemente porque esto pueda llevar a una sobreprotección que dificulte la relación con los amigos. Dichos estudios no son específicos de supervivientes de TSNC.

El estilo de resolución de problemas, en cambio, y según el presente estudio, no determina la aceptación social y sí lo hace el uso de un estilo improductivo. Esto parece lógico si tenemos en cuenta que este estilo se basa en la autoinculpación, el pensamiento ilusorio o la preocupación excesiva, entre otras características, lo cual puede dar lugar al rechazo por parte de los iguales. La disminución de calidad de vida en esta dimensión psicosocial causada por el estilo improductivo del adolescente podría, sin embargo, verse compensada cuando los padres actúan utilizando un estilo de afrontamiento de búsqueda de ayuda interpersonal, tal como indican nuestros resultados, que señalan una relación directa entre la aceptación social y el uso de ese estilo de afrontamiento. Al margen de ello, cabría pensar que si el adolescente utiliza un estilo de afrontamiento basado en la relación con los demás, la aceptación social mejoraría, pero nuestros datos no confirman esta relación. Es más, existen algunos estudios que sugieren que el uso de un perfil de afrontamiento de estilo represivo (esto es, basado en una restricción de la interacción social, o en un uso de la misma en el que no se busca compartir los problemas) se relaciona con una mejor CVRS en el área psicosocial ${ }^{(47,48)}$. 
Este conjunto de resultados permite especular con la posibilidad de que los supervivientes de TC/TSNC desarrollen un estilo propio de afrontamiento basado en una actitud defensiva ${ }^{(45)}$ en la que se encubrirían las dificultades de la enfermedad tendiendo a negarlas y a construir así un estilo represivo que facilitaría la adaptación al entorno social ${ }^{(22,49)}$. Sin embargo, este estilo conllevaría el problema de que podría dar lugar a un mayor malestar psicológico ${ }^{(48)}$.

Nuestros resultados confirman parcialmente el posible papel modulador del CI sobre los aspectos psicosociales de la calidad de vida, ya que únicamente la velocidad de procesamiento se relaciona con el Entorno Escolar y sólo en la versión proxy. No podemos considerar que esta falta de relación se deba a que nuestra muestra presente menos déficits en el CI que los observados en otros trabajos, ya que nuestros resultados coinciden con los de otros estudios al señalar que el grupo de supervivientes de TC/TSNC presenta significativamente mayores déficits en todas las áreas cognitivas evaluadas en comparación de los supervivientes de otras neoplasias ${ }^{(50)}$. Además, estarían en concordancia con los datos aportados por el meta-análisis realizado por Robinson y colaboradores ${ }^{(51)}$, que examina a 1.318 niños tratados de TC, provenientes de 39 estudios. Dicho trabajo señala que la mayoría de supervivientes obtienen un CI con una desviación típica por debajo de la media, experimentando déficits clínicos significativos. En los resultados obtenidos en la presente investigación en las cuatro áreas evaluadas (CV, RP, MT, VP), más del 50\% de participantes presentan CI por debajo de una desviación típica de la puntuación media. Por lo tanto, parece que nuestros resultados apuntan a que la calidad de vida en los aspectos psicosociales, sólo está mediada por el CI cuando dichos aspectos se refieren al ámbito escolar y no a otros ámbitos de la vida del adolescente. Dado que esto se ha observado solamente en la versión proxy, sorprende que los padres no perciban más relaciones entre la CVRS y funciones cognitivas en referencia a sus hijos. Una posible explicación estarían en concordancia con lo expuesto por Maddrey et al. ${ }^{(4)}$ en referencia a que los padres con hijos supervivientes de TC/SNC en ocasiones sobrevaloran las capacidades neurocognitivas de éstos, mostrando desacuerdo entre su percepción y los datos objetivos.

Nuestros datos no confirman la posible relación entre el malestar parental y los aspectos psicosociales de la calidad de vida, pese a que ésta sí se ha observado en muestras de supervivientes de tumores no-TC/TSNC ${ }^{(22)}$ aunque sólo en la dimensión de Aceptación Social. Podría ocurrir que en supervivientes de tumores TC/SNC no se dé esta relación ni en los aspectos psicosociales ni tampoco en otras dimensiones de la CVRS, ya que hay datos de otros trabajos que así lo indican ${ }^{(52)}$. Por otra parte, en nuestra muestra, y según los resultados de la escala PSS, el porcentaje de progenitores que muestra sintomatología de estrés es solo del 20\%. Sería necesario realizar más investigaciones con muestras más amplias de adolescentes supervivientes de tumores TC/SNC antes de asumir de forma definitiva que el malestar parental no influye en la calidad de vida de estos adolescentes.

El planteamiento de este trabajo no pretendía descartar el posible papel modulador sobre la calidad de vida de otros factores como las secuelas, el tipo de tratamiento recibido, el tiempo transcurrido desde el diagnóstico, o la edad en el diagnóstico, y nuestros datos confirman que estos factores sí se relacionan con la calidad de vida en los aspectos psicosociales, si bien con diferencias entre las versiones auto y proxy. Los años transcurridos desde el diagnóstico afectan negativamente a la dimensión Amigos y Apoyo Social en ambas versiones, 
confirmando así lo observado en otras investigaciones ${ }^{(10,19)}$. Las secuelas y el haber recibido radioterapia sólo afectan a las valoraciones en la versión proxy y en el caso de la radioterapia, de una manera muy marginal, ya que la varianza explicada es inferior al $10 \%$. Esto lleva a pensar que los progenitores atribuyen la calidad de vida de los aspectos psicosociales del adolescente superviviente más a factores clínicos, poco modificables, que a aspectos relacionados con el comportamiento y las vivencias (estrategias de afrontamiento, malestar emocional) que podrían ser tratados mediante intervenciones terapéuticas adecuadas. Finalmente, el que exista una relación directa entre la edad de diagnóstico y una mejor calidad de vida en la dimensión Relación con los padres y vida familiar contradice la literatura previa $^{(3,18)}$, pero podría explicarse considerando que a edades más avanzadas la vinculación familiar y la relación con los padres está mejor establecida y permite superar mejor las consecuencias derivadas de la enfermedad y los tratamientos, si bien esto debería corroborarse en estudios posteriores.

El conjunto de resultados aportado confirma que las estrategias de afrontamiento modulan la calidad de vida del adolescente en los aspectos psicosociales y, en menor medida, que también lo hace el CI. Sin embargo, estos resultados se han obtenido con algunas limitaciones de las que deseamos dejar constancia. Por un lado, la muestra es relativamente escasa y, por otro lado, en el caso de los progenitores sólo se ha tenido en cuenta a las madres. Respecto a la primera limitación, es posible que estudios con muestras más amplias pudieran detectar posibles efectos producidos por las secuelas y los bajos niveles del CI que en esta investigación no han sido confirmados con los análisis realizados. En lo concerniente a la segunda limitación, sería necesario confirmar si las estrategias de afrontamiento desarrolladas por las madres son también utilizadas por los padres y si estos juegan un papel similar o diferente al de las madres en la modulación de la CVRS del superviviente.

Sin perder de vista dichas limitaciones, podemos decir que el conjunto de resultados obtenidos en la presente investigación confirmaría la importancia de las estrategias de afrontamiento del adolescente y de los padres para la calidad de vida psicosocial, cuando la misma es valorada por el propio adolescente. Por lo tanto, parece adecuado plantear que el estilo de resolución de problemas, en el caso de los adolescentes, y de búsqueda de ayuda, en el caso de los progenitores, debe ser potenciado por parte de las personas encargadas del cuidado de estos supervivientes. Finalmente, y pese a que nuestros resultados no permiten afirmar que las limitaciones cognitivas de un bajo CI afecten a la CVRS, debería seguir investigándose este aspecto, ya que no podemos descartar completamente que un bajo CI pueda afectar a las relaciones sociales con los iguales.

\section{Conflictos de interés}

Los autores del presente trabajo manifiestan no tener ningún conflicto de intereses.

\section{Agradecimientos}

A todos los participantes que colaboraron desinteresadamente en el presente trabajo. 


\section{Referencias bibliográficas}

1. Oeffinger KC, Mertens AC, Sklar CA, Kawashima T, Hudson MM, Meadows AT, et al. Chronic health conditions in adult survivors of childhood cancer. N Engl J Med 2006;355:1572-82. doi: 10.1056/NEJMsa060185

2. Gurney JG, Krull KR, Kadan-Lottick N, Nicholson HS, Nathan PC, Zebrack B, et al. Social outcomes in the Childhood Cancer Survivor Study Cohort. J Clin Oncol 2009;27:2390-5. doi: $10.1200 /$ JCO.2008.21.1458

3. Aarsen FK, Paquier PF, Reddingius RE, Streng IC, Arts W-FM, Evera-Preesman M, et al. Functional outcome after low-grade astrocytoma treatment in childhood. Cancer 2006;106:396-402.

4. Maddrey AM, Bergeron JA, Lombardo ER, McDonald NK, Mulne AF, Barenberg PD, et al. Neuropsychological performance and quality of life of 10 year survivors of childhood medulloblastoma. J Neurooncol 2005;72:245-53. doi: 10.1007/s11060-004-3009-Z

5. Rueegg CS, Gianinazzi ME, Rischewski J, Beck Popovic M, von der Weid NX, Michel $\mathrm{G}$, et al. Health-related quality of life in survivors of childhood cancer: the role of chronic health problems. J Cancer Surviv 2013;7:511-22. doi: 10.1007/s11764-013-0288-4

6. Zeltzer LK, Recklitis C, Buchbinder D, Zebrack B, Casillas J, Tsao JCI, et al. Psychological status in childhood cancer survivors: A report from the childhood cancer survivor study. J Clin Oncol. 2009;27:2396-404. doi: 10.1002/pon.1959

7. Cardarelli C, Cereda C, Masiero L, Viscardi E, Faggin R, Laverda A, et al. Evaluation of health status and health-related quality of life in a cohort of Italian children following treatment for a primary brain tumor. Pediatr Blood Cancer 2006;46:637-44. doi. org/10.1002/pbc. 20480

8. Chan CWH, Choi KC, Chien WT, Cheng KKF, Goggins W, So WKW, et al. Healthrelated quality-of-life and psychological distress of young adult survivors of childhood cancer in Hong Kong: Health-related quality of life. Psychooncology 2014;23:229-36. doi: 10.1007/s11136-017-1716-0

9. Maunsell E, Pogany L, Barrera M, Shaw AK, Speechley KN. Quality of life among longterm adolescent and adult survivors of childhood cancer. J Clin Oncol 2006;24:2527-35. doi: 10.1200/JCO.2005.03.9297

10. Pérez-Campdepadrós M, Castellano-Tejedor C, Sábado-Álvarez C, Gros-Subías L, Capdevila L, Blasco-Blasco T. Type of tumour, gender and time since diagnosis affect differently health-related quality of life in adolescent survivors: Type of tumour and adolescents' health-related quality of life. Eur J Cancer Care 2015;24:635-41. doi: 10.1111/ecc. 12215

11. Pogorzala M, Styczynski J, Kurylak A, Debski R, Wojtkiewicz M, Wysocki M. Healthrelated quality of life among paediatric survivors of primary brain tumours and acute leukaemia. Qual Life Res 2010;19:191-8. doi: 10.1007/s11136-009-9580-1

12. Speechley KN, Barrera M, Shaw AK, Morrison HI, Maunsell E. Health-Related Quality of Life Among Child and Adolescent Survivors of Childhood Cancer. J Clin Oncol 2006;24:2536-43. doi: 10.1200/JCO.2005.03.9628

13. Stam H, Grootenhuis MA, Caron HN, Last BF. Quality of life and current coping in young adult survivors of childhood cancer: positive expectations about the further course of the disease were correlated with better quality of life. Psychooncology 2006;15:31-43. doi: 10.1002/pon.920 
14. Zebrack BJ, Landier W. The perceived impact of cancer on quality of life for posttreatment survivors of childhood cancer. Qual Life Res. 2011;20:1595-608. doi: 10.1007/ s11136-011-9893-8

15. Reimers TS, Mortensen EL, Nysom K, Schmiegelow K. Health-related quality of life in long-term survivors of childhood brain tumors. Pediatr Blood Cancer 2009;53:1086-91. doi: $10.1002 / p b c .22122$

16. Zuzak TJ, Poretti A, Drexel B, Zehnder D, Boltshauser E, Grotzer MA. Outcome of children with low-grade cerebellar astrocytoma: long-term complications and quality of life. Childs Nerv Syst 2008;24):1447-55. doi: 10.1007/s00381-008-0692-7

17. Frange P, Alapetite C, Gaboriaud G, Bours D, Zucker JM, Zerah M, et al. From childhood to adulthood: long-term outcome of medulloblastoma patients. The Institut Curie experience (1980-2000). J Neurooncol 2009;95:271-9. doi: 10.1007/s11060-009-9927-z.

18. Sands SA, Pasichow KP, Weiss R, Garvin J, Gardner S, Dunkel IJ, et al. Quality of life and behavioral follow-up study of Head Start I pediatric brain tumor survivors. J Neurooncol 2011;101:287-95. doi: 10.1007/s11060-010-0260-3

19. Bath SR, Goodwin TL, Burwinkle TM, Lansdale MF, Dahl GV, Huhn SL, et al. Profile of daily life in children with brain tumours: An assessment of health-related quality of life. J Clin Oncol 2005;23:5493-500. doi: 10.1200/JCO.2005.10.190

20. Benesch M, Spiegl K, Winter A, Passini A, Lackner H, Moser A, et al. A scoring system to quantify late effects in children after treatment for medulloblastoma/ependymoma and its correlation with quality of life and neurocognitive functioning. Childs Nerv Syst 2009;25:173-81. doi: 10.1007/s00381-008-0742-1

21. Huang I-C, Brinkman TM, Kenzik K, Gurney JG, Ness KK, Lanctot J, et al. Association between the prevalence of symptoms and health-related quality of life in adult survivors of childhood cancer: a report from the St Jude Lifetime Cohort Study. J Clin Oncol 2013;31:4242-51. doi: 10.1200/JCO.2012.47.8867

22. Castellano-Tejedor C, Pérez-Campdepadrós M, Capdevila L, Blasco-Blasco T. Surviving cancer: The psychosocial outcomes of childhood cancer survivors and its correlates. J Health Psychol 2016;21:1491-502. doi: 10.1177/1359105314557503.

23. Barakat LP, Daniel LC, Smith K, Renée Robinson M, Patterson CA. Parental ProblemSolving Abilities and the Association of Sickle Cell Disease Complications with Health-Related Quality of Life for School-Age Children. J Clin Psychol Med Settings 2014;21:56-65. doi: 10.1007/s10880-013-9379-7

24. Fuemmeler BF, Mullins LL, Marx BP. Posttraumatic stress and general distress among parents of children surviving a brain tumor. Child Health Care. 2001;30:169-82.

25. Bruce M, Gumley D, Isham L, Fearon P, Phipps K. Post-traumatic stress symptoms in childhood brain tumour survivors and their parents: Coping strategies and parent child interactions. Child Care Health Dev 2011;37:244-51. doi: 10.1111/j.13652214.2010.01164.x

26. Forinder U, Norberg AL. Posttraumatic growth and support among parents whose children have survived stem cell transplantation. J Child Health Care 2014;18:326-35. doi: $10.1177 / 1367493513496666$

27. Hovén E, von Essen L, Norberg AL. A longitudinal assessment of work situation, sick leave, and household income of mothers and fathers of children with cancer in Sweden. Acta Oncol. 2013;52:1076-85. doi: 10.3109/0284186X.2012.760846

28. Castellano C, Blasco T, Oller A, Pérez-Campdepadrós M, Sánchez de Toledo J, Capdevila L. Calidad de vida en adolescentes supervivientes de cáncer infanto-juvenil. Med Clin 2009;133:783-6. doi: 10.1016/j.medcli.2009.04.034 
29. Ness KK, Gurney JG. Adverse Late Effects of Childhood Cancer and Its Treatment on Health and Performance. Annu Rev Public Health 2007;28:279-302. doi: 10.1146/ annurev.publhealth.28.021406.144049

30. Ravens-Sieberer U, Gosch A, Rajmil L, Erhart M, Bruil J, Duer W, et al. KIDSCREEN-52 quality-of-life measure for children and adolescents. Expert Rev Pharmacoecon Outcomes Res 2005;5:353-64. doi: 10.1586/14737167.5.3.353

31. Aymerich M, Berra S, Guillamón I, Herdman M, Alonso J, Ravens-Sieberer U et al . Development of the Spanish version of the KIDSCREEN: a health-related quality of life instrument for children and adolescents. 2005;19:93-102.

32. Frydenberg E, Lewis R. Boys play sport and girls turn to others: age, gender and ethnicity as determinants of coping. J Adolesc 1993;16:253-66. doi: 10.1006/jado.1993.1024

33. Frydenberg E, Lewis R. A Replication study of the structure of the Adolescent Coping Scale: Multiple forms and applications of a self-report inventory in a counselling and research context. Eur J Psychol Assess 1996;12:224-35.

34. Pereña J, Seisdedos N. Manual ACS: escalas de afrontamiento para adolescentes. Madrid: Ediciones TEA. TEA. 1996.

35. Wechsler, D. Manual for the Wechsler preschool and primary scale of intelligence revised. Psychological Corporation., New York. 1989.

36. Wechsler, D. Escala de Inteligencia de Wechsler para adultos-III. Adaptación española: Departamento de I+D. TEA Ediciones, S.A. (2a edición revisada). Madrid: Ediciones TEA. 2001.

37. Wechsler, D. Medida de la inteligencia para adultos. WAIS-III. Buenos Aires: Paidós. 2002.

38. Wechsler, D. Escala de Inteligencia de Wechsler para niños-IV. (1 ${ }^{\text {a }}$ edición). Madrid: TEA. 2005.

39. Crespo M, Cruzado JA. La evaluación del afrontamiento: adaptación española del cuestionario COPE con una muestra de estudiantes universitarios. Análisis y Modificación de la Conducta 1997;23:797-830.

40. Gutiérrez F, Peri JM, Torres X, Caseras X, Valdés M. Three dimensions of coping and a look at their evolutionary origin. J Res Personal 2007;41:1032-53. doi: 10.1016/j. jrp.2007.01.006

41. Cohen S, Kamarck T, Mermelstein RA. A global measure of perceived stress. J.Health Soc Behav 1983;24:385-96.

42. Remor E. Psychometric properties of the European Spanish version of the Perceived Stress Scale (PSS). Spanish J Psychol 2006;9:86-93.

43. Castellano C, Sánchez de Toledo J, Blasco T, Gros L, Capdevila L Pérez-Campdepadrós M. Afrontamiento y malestar emocional parental en relación a la calidad de vida del adolescente oncológico en remisión. Psicooncología 2010; 7: 415-31.

44. Grootenhuis MA, Last BF. Children with cancer with different survival perspectives: defensiveness, control strategies, and psychological adjustment. Psychooncology 2001;10:305-14. doi: 10.1002/pon.529

45. Phipps S. Adaptive Style in Children with Cancer: Implications for a Positive Psychology Approach. J Pediatr Psychol 2007;32:1055-66. doi: 10.1093/jpepsy/jsm060

46. Aldridge AA, Roesch SC. Coping and Adjustment in Children with Cancer: A MetaAnalytic Study. J Behav Med 2007;30:115-29. doi: 10.1007/s10865-006-9087-y

47. Eiser C, Greco V, Vance YH, Horne B, Glaser A. Perceived discrepancies and their resolution: quality of life in survivors of childhood cancer. Psychol Health. 2004;19:1528. doi.org/10.1080/08870440310001594501 
48. Turner-Sack AM, Menna R, Setchell SR. Posttraumatic growth, coping strategies, and psychological distress in adolescent survivors of cancer. J Pediatr Oncol Nurs 2012;29:709. doi: 10.1177/1043454212439472

49. Erickson SJ, Gerstle M, Montague EQ. Repressive adaptive style and self-reported psychological functioning in adolescent cancer survivors. Child Psychiatry Hum Dev 2008;39:247-60. doi: 10.1007/s10578-007-0085-2

50. Ellenberg L, Liu Q, Gioia G, Yasui Y, Packer RJ, Mertens A, et al. Neurocognitive status in long-term survivors of childhood CNS malignancies: A report from the Childhood Cancer Survivor Study. Neuropsychol 2009;23:705-17. doi: 10.1037/a0016674

51. Robinson KE, Kuttesch JF, Champion JE, Andreotti CF, Hipp DW, Bettis A, et al. A quantitative meta-analysis of neurocognitive sequelae in survivors of pediatric brain tumors. Pediatr Blood Cancer 2010;55:525-31. doi: 10.1002/pbc.22568

52. Pérez-Campedpadrós M. Variables moduladoras de la calidad de vida en supervivientes pediátricos de tumores en el Sistema Nervioso Central. Tesis Doctoral. Bellaterra, Barcelona, 2015. 